\title{
Article \\ Organoprotective Effects of Spironolactone on Top of Ramipril Therapy in a Mouse Model for Alport Syndrome
}

\author{
Diana Rubel ${ }^{1,+}$, Yanqin Zhang ${ }^{1,2, \dagger}$, Nenja Sowa ${ }^{1}$, Rainer Girgert ${ }^{1}$ and Oliver Gross ${ }^{1, *(\mathbb{D})}$ \\ 1 Clinic of Nephrology and Rheumatology, University Medical Center Goettingen, 37075 Goettingen, Germany; \\ diana@rubel.de (D.R.); yiran6666@126.com (Y.Z.); nenjasowa@googlemail.com (N.S.); \\ rainer.girgert@med.uni-goettingen.de (R.G.) \\ 2 Department of Pediatrics, Peking University First Hospital, Beijing 100034, China \\ * Correspondence: gross.oliver@med.uni-goettingen.de; Tel.: +49-551-60488 \\ + These authors contributed equally to this work.
}

Citation: Rubel, D.; Zhang, Y.; Sowa,

N.; Girgert, R.; Gross, O.

Organoprotective Effects of

Spironolactone on Top of Ramipril

Therapy in a Mouse Model for Alport

Syndrome. J. Clin. Med. 2021, 10, 2958

https://doi.org/10.3390/

jcm10132958

Academic Editors:

Emmanuel Andrès,

Smaragdi Marinaki and

Sophia Lionaki

Received: 16 May 2021

Accepted: 29 June 2021

Published: 30 June 2021

Publisher's Note: MDPI stays neutral with regard to jurisdictional claims in published maps and institutional affiliations.

Copyright: (c) 2021 by the authors. Licensee MDPI, Basel, Switzerland. This article is an open access article distributed under the terms and conditions of the Creative Commons Attribution (CC BY) license (https:// creativecommons.org/licenses/by/ $4.0 /)$.

\begin{abstract}
Angiotensin-converting enzyme inhibitors (ACEi) delay progression of the inherited renal disease Alport syndrome. However, the effect of ACEis weakens gradually due to an "aldosterone escape". Here, we investigate if an aldosterone antagonist can counteract loss of ACEi-efficacy. COL4A3-/- mice were treated with ramipril (ACEi), starting at 4.5 weeks of age, and spironolactone was added at 7 weeks of age. Lifespan until renal failure, as well as kidney function parameters, were investigated. Dual therapy decreased proteinuria levels compared to ACEi monotherapy. Matrix accumulation, as well as tubulointerstitial and glomerular scar-tissue formation, were significantly reduced compared to untreated mice and ACEi-monotherapy at 75 and 100 days. Lifespan in dual treated mice was extended compared to untreated mice. However, lifespan was not superior to ACEi monotherapy-despite improved urea-nitrogen levels in the dual therapy group. In conclusion, adding the aldosterone-antagonist spironolactone to ACEi therapy further improved kidney function and reduced proteinuria and fibrosis. However, survival was not improved further, possibly due to premature death from side effects of dual therapy such as hyperkalemia. Thus, dual therapy could offer an effective therapy option for Alport syndrome patients with progressive proteinuria. However, the risks of adverse events require close monitoring.
\end{abstract}

Keywords: hereditary kidney disease; progressive kidney fibrosis; nephroprotective therapy; type IV collagen

\section{Introduction}

Alport syndrome (AS) is an inherited renal disease caused by variants in COL4A3, COL4A4, or COL4A5 gene [1-3]. The clinical manifestations of Alport syndrome include hematuria, proteinuria, and progressive renal failure, sometimes accompanied by hearing loss and ocular lesions [4]. End-stage renal disease (ESRD) is inevitable in the majority of patients with Alport syndrome and typically occurs during early adulthood. It is reported that $90 \%$ of patients develop ESRD by the age of 30 years in males with X-linked Alport syndrome (XLAS) due to variants in COL4A5 gene. In autosomal recessive Alport syndrome (ARAS) with variants in the COL4A3 or COL $4 A 4$ genes, female and male patients are equally affected and also reach ESRD early in life [5-9].

In the last two decades, potential therapies preventing or delaying the progression of kidney dysfunction of patients with AS have become a prominent focus of research. Most relevant, blockade of the renin-angiotensin-aldosterone system (RAAS) by angiotensinconverting enzyme inhibitors (ACEi) or angiotensin receptor blockade (ARB) has been shown to delay renal failure and reduce renal fibrosis in COL4A3 knockout mice as animal models of AS [10,11]. Moreover, retrospective analysis of patients with AS strongly suggested that ACEi therapy can delay ESRD by many years [12]. Retrospective data from children with AS showed that proteinuria decreased significantly in the first two years 
of ACEi and ARB therapy [13,14]. Early RAAS-blockade does not only improve renal survival in patients with AS, but also increases the hope for finding other drugs which work additive to ACEis to further delay disease progression in AS.

In the mouse model for Alport syndrome, some of the positive effects reducing the amount of proteinuria and serum urea of ramipril therapy were lost after several weeks of treatment [10]. Similarly, retrospective data from children with AS indicate that proteinuria gradually increases after three years of ACEi therapy [14]. This negative phenomenon is similar to the ESCAPE trial with aggressive blood pressure control by ramipril in children with chronic kidney disease (CKD), in which some of the early antiproteinuric effects was lost by the third year of follow-up [15]. Experimental data suggest that $30-40 \%$ of patients with CKD develop increased levels of plasma aldosterone whilst on long-lasting of ACEi therapy, known as "aldosterone escape", which correlates with the decline of glomerular filtration rate $[16,17]$.

As a consequence, the "aldosterone escape" is hypothesized to be an important negative pathomechanism for disease progression in AS in the course of long-lasting ACEinhibition. In the present study, we investigate the hypothesis if the aldosterone antagonist spironolactone can counteract this loss of ACEi-efficacy in the Alport mouse model.

\section{Materials and Methods}

\subsection{Animals and Treatment}

Heterozygous COL4A3 knockout mice (JAX stock \#002908, Jackson Laboratories, Bar Harbor, ME, USA) were crossbred on a 129/SvJ genetic background. DNA was isolated from tail biopsies according to manufacturer instruction using Nucleo Spin ${ }^{\circledR}$ Tissue Kit (Macherey Nagel, Düren, Germany). Genotyping of the mice was carried out by PCR with the TopTaq ${ }^{\circledR}$ DNA Polymerase (Qiagen, Hilden, Germany) using a combination of three different Primer (COL4A3 forward 5'-CCAGGCTTAAAGGGAAATCC-3'; COL4A3 reverse 5'-CCTGCTAATATAGGGTTCGAGA-3'; COL4A3 mutant 5'-AAT CGC CAA TGA CAA GAC G-3'). Treatment protocols for the mice were approved by local German authorities and supervised by veterinarians. Sixteen $\mathrm{COL} 4 A 3-/-$ mice per group were treated with ramipril (Sanofi-Aventis, Paris, France) (ACEi-group) or ramipril plus spironolactone (Sigma Aldrich, S3378, St. Louis, MO, USA). (ACEi+Spiro). Medication was given orally via the drinking water. Ramipril was started in both groups at 4.5 weeks of age with a daily dose of $10 \mathrm{mg} / \mathrm{kg} /$ day during the whole experiment. Spironolactone was added to ramipril in the ACEi+Spiro group at 7 weeks of age with a daily dose of $50 \mathrm{mg} / \mathrm{kg} /$ day.

Three to four mice per group were sacrificed at two different stages of the disease (75 and 100 days). In the remaining 8 to 9 mice per group, lifespan until renal failure was monitored. Animals were weighed on a weekly basis, and state of health was monitored daily. All results were compared to wildtype mice (WT) and untreated COL4A3-/mice (Plac).

\subsection{Kidney Function Parameters}

Proteinuria was measured in spontaneous urine. Urine samples were processed via chloroform-methanol precipitation and separated by SDS-PAGE using 4-12\% Novex Tris Glycine polyacrylamide gradient gels (Life Technologies, Carlsbad, CA, USA). The net intensity of protein bands was measured after Coomassie staining by densitometry using Kodak 1D software. Blood urea nitrogen (BUN) was analyzed using serum samples of sacrificed mice (kinetic UV-Test, Cobas8000 Modular Analyzer Series, Roche Diagnostics, Mannheim, Germany). Serum aldosterone levels were measured by an aldosterone ELISA (catalog no. RE52301, IBL International GmbH, Hamburg, Germany) according to manufacturer instructions.

\subsection{Immunohistology}

Kidneys of sacrificed mice were kept in $4 \%$ paraformaldehyde at $40 \mathrm{C}$ overnight and immersion-fixed using HistoCore PEARL (Leica, Wetzlar, Germany). Paraffin-embedded 
kidneys were sectioned at $3 \mu \mathrm{m}$ on a Reichert-Jung 2040 Autocut Microtome (Leica Microsystems, Wetzlar, Germany). For staining of laminin 111 and fibronectin, the slides were de-paraffinized and rehydrated. Sections were incubated overnight at $4{ }^{\circ} \mathrm{C}$ with the primary antibodies rabbit anti-laminin 111 (1:25, Ab11575, Abcam, Cambridge, UK), and goat anti-fibronectin (1:50, sc6952, Santa Cruz Tech, Dallas, TX, USA). As a negative control, slides were incubated with blocking solution ( $5 \%$ donkey serum in Tris-buffered saline). After washing the samples were stained with the secondary antibody. The Alexa 488-labled antibody goat anti-rabbit (A11008, Lifetech, Waltham, MA, USA) was used to prepare the laminin 111 staining and the donkey anti-goat (A11055, Lifetech, Waltham, MA, USA) was added to the slides for fibronectin staining. Both antibodies incubated for one hour at room temperature in the dark. After washing all slides were mounted in fluorescence mounting medium (S303, Dako, Waldbronn, Germany).

\subsection{Scoring of Immunohistology}

For the glomerular score, images were taken at $400 \times$ magnification. For the tubulointerstitial score, a $100 \times$ magnification (laminin 111) and $200 \times$ magnification (fibronectin) were used. Six images per kidney of three mice per group were scored by three independent individuals in a blinded manner, as previously described [10,11] A pre-defined scoring system from zero (best, normal glomerular or tubular structure) to 3 (worst, severe glomerulosclerosis or tubulointerstitial damage) were used for classification.

\subsection{Statistical Analysis}

Results are presented as mean and standard deviation (SD). One-tailed student T test was used for analyzing differences in body weight. All other data throughout the manuscript were analyzed by two-way analysis of variance (ANOVA) followed by Bonferroni's multiple comparison test.

\section{Results}

In the present study, we investigate the hypothesis if the aldosterone antagonist spironolactone can counteract this loss of ACEi-efficacy in the Alport mouse model.

\subsection{Effect on Aldosterone Levels}

Untreated mice (Plac) showed very high levels of serum aldosterone $(2622 \mathrm{pg} / \mathrm{mL}$; Figure 1). In the ACEi group, serum aldosterone decreased to $417 \pm 158 \mathrm{pg} / \mathrm{mL}$ at 75 days of age and rose to $895 \pm 252 \mathrm{pg} / \mathrm{mL}$ at 100 days, mimicking an "aldosterone-escape" described in literature $[16,17]$. When spironolactone was added on top of ramipril, serum aldosterone decreased to $233 \pm 62 \mathrm{pg} / \mathrm{mL}$ at 75 days and $312 \pm 82 \mathrm{pg} / \mathrm{mL}$ at 100 days These levels were in a low range comparable to wild type mice $(211 \pm 107 \mathrm{pg} / \mathrm{mL})$ and indicated that dual RAAS-blockade (ACEi+Spiro) was effective to test our hypothesis in the following experiments: 


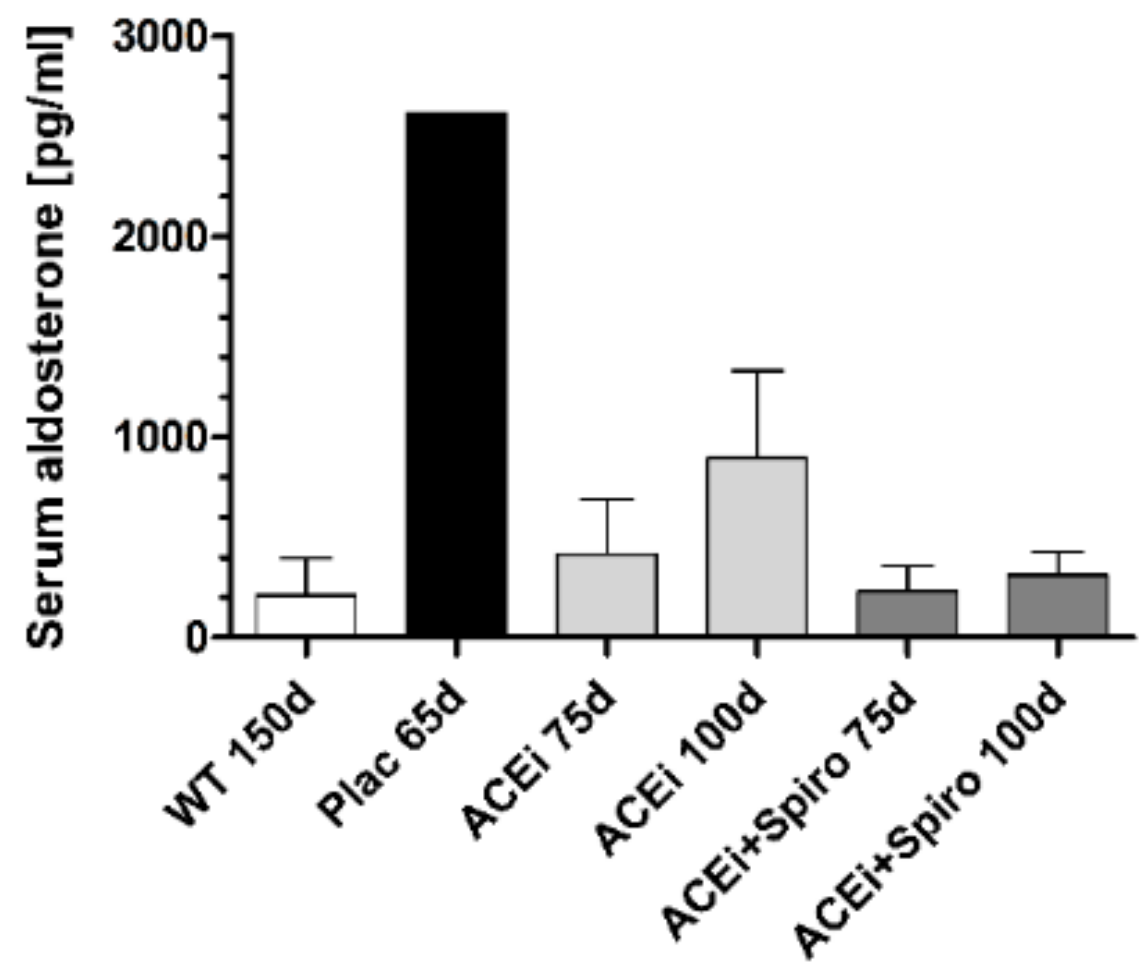

Figure 1. Serum aldosterone levels indicating an aldosterone-escape in Alport mice receiving Angiotensin-converting enzyme inhibitor (ACEi)-monotherapy.

\subsection{Effect on Proteinuria and Kidney Function Parameters}

Kidney function (BUN and proteinuria) was monitored in a total of 17 COL4A3- / mice (4 untreated (Plac), 7 ramipril monotherapy (ACEi), and 6 with dual therapy (ACEi+Spiro)). Differences in proteinuria were not statistically evaluated, because of the semi-quantitative method used and the limited number of mice per group. In ACEi treated mice, urinary protein excretion at 50 days of age was much lower compared to the untreated group (Plac) at 65 days (Figure 2A). However, ACEi monotherapy was not able to maintain its antiproteinuric effect: levels of proteinuria constantly rose despite ACEi therapy at 75 and 100 days of age to levels similar to Alport mice in the placebo group at 65 days. In contrast, dual RAAS-blockade by adding spironolactone on top of ACEi was more effective in preserving the antiproteinuric effect on urinary relative protein excretion (Figure 2A, ACEi+Spiro). Further analysis of the relative amounts of urinary protein showed that albumin excretion levels were comparable in both treated groups compared to placebo group (Figure 2B). In mice treated with spironolactone on top of ramipril, excretion of high molecular weight proteins and low molecular weight proteins was reduced compared to placebo and ACEi monotherapy during disease progression (Figure 2B). High molecular weight proteins such as immunoglobulins account for glomerular damage or leakage and low molecular weight proteins such as microglobulins are a sign for tubular damage. In conclusion, dual RAAS-blockade by adding spirolonactone on top of ACEi appeared to have a greater potential in preserving glomerular and tubular function in our mice during the course of AS. 

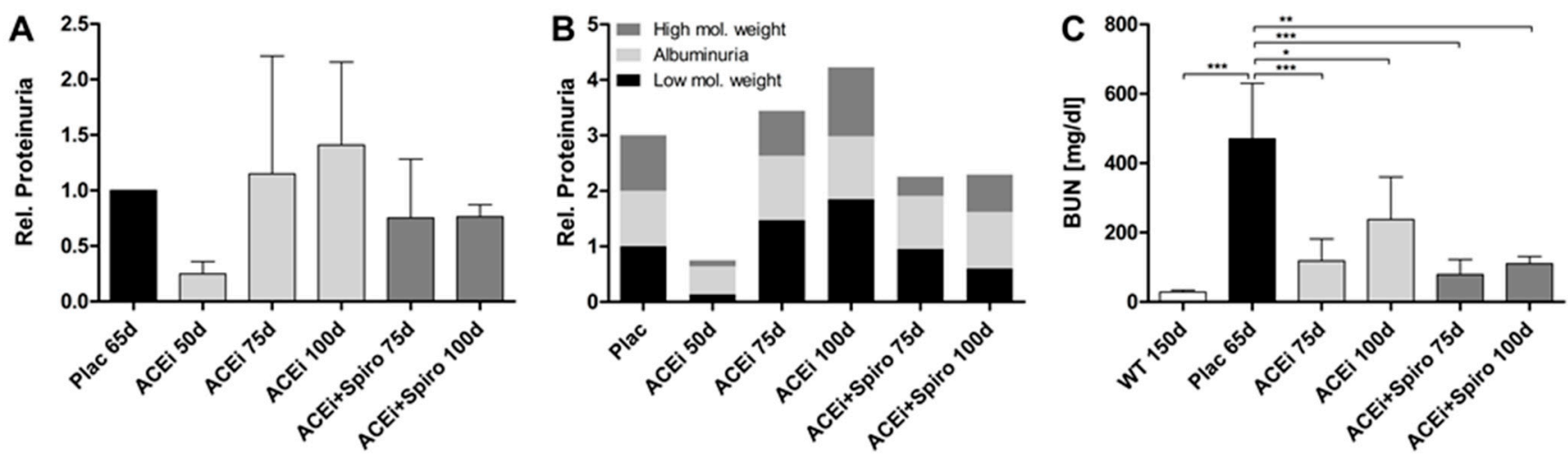

Figure 2. Effect on protein excretion and kidney function on days 50, 75 and 100 of therapy with ACEi and ACEi+Spiro. (A) Total renal protein excretion (relative to untreated mice at 65 days) in the ACEi group compared to the ACEi+Spiro group ( $n=3$ for each group); (B) Relative change in excretion of high molecular weight proteins (glomerular damage), albumin and low molecular weight proteins (tubular damage) in the ACEi group compared to the ACEi+Spiro group ( $n=3$ for each group); (C) Blood urea nitrogen (BUN) levels in the untreated mice compared to the ACEi group and the ACEi+Spiro group ( $n=3$ for WT and ACEi 100d, $n=4$ for Plac, ACEi $75 \mathrm{~d}$ and ACEi+Spiro $75 \mathrm{~d}, n=2$ for ACEi+Spiro 100d); *** $p<0.001 ;{ }^{* *} p<0.01 ; * p<0.05$.

Untreated mice (Plac) showed a remarkable increase in their BUN levels at 65 days, representing the very advanced stage of AS close to end-stage renal failure (Figure 2C). Ramipril monotherapy decreased the BUN levels significantly at 75 and 100 days (compared vs. placebo). Add on therapy with spironolactone on top of ramipril decreased BUN even further, but not significantly different to ACEi-monotherapy, at 75 days and also at 100 days of age (Figure 2C). In the ACEi+Spiro group, two mice died prematurely prior to BUN measurement on day 100, which might reflect an increased risk for hyperkalemia in this group. In conclusion, dual RAAS-blockade appeared to preserve the kidney's detoxification function better than ACEi monotherapy.

\subsection{Effect on Matrix Accumulation and Fibrosis during Pathogenesis of Alport Syndrome}

Tubulointerstitial and glomerular matrix accumulation (laminin 111) was significantly increased in ACEi-treated and untreated Alport mice (Figure 3B-D) compared to wild type mice (Figure $3 \mathrm{~A} ; p<0.001$ ). In contrast, when spironolactone was added on top of ramipril, tubulointerstitial matrix accumulation was significantly improved at 75 days compared to ACEi treated mice $(p<0.01)$, an effect which was not maintained until day 100 (Figure 3G). The glomerular extracellular matrix accumulation improved significantly by dual RAAS blockade (ACEi+Spiro; Figure 3E,F) compared to untreated Alport mice at 75 and 100 days of age $(p<0.05$ and $p<0.01)$ (Figure 3H). In conclusion, dual RAASblockade appeared to have a greater inhibitory effect on excessive matrix production in AS than ACEi monotherapy. 

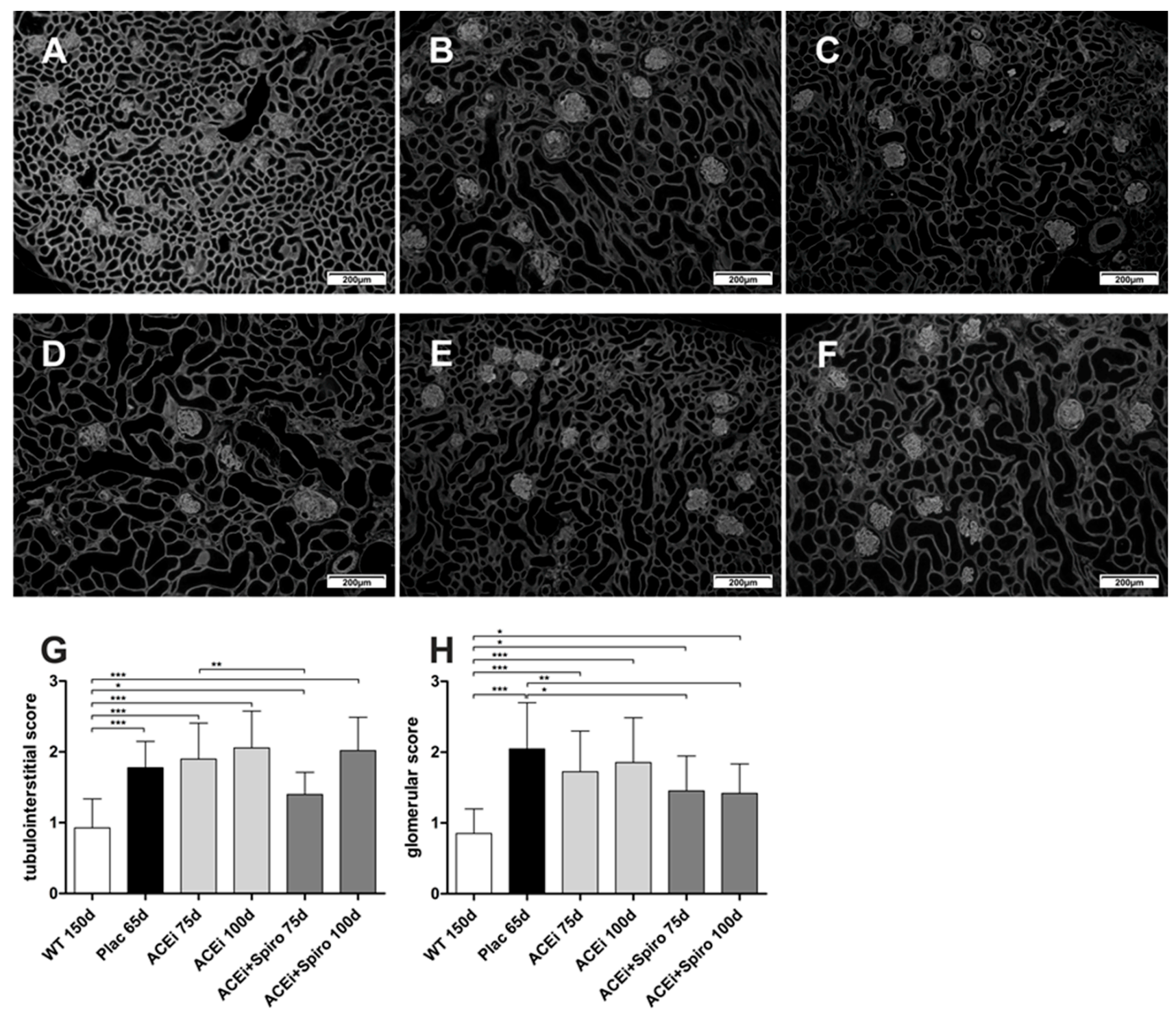

Figure 3. Therapeutic effect on matrix accumulation during pathogenesis of Alport Syndrome (AS). Immune-fluorescence staining of laminin 111, magnification 100-fold. (A) wildtype control; (B) Alport mice, ACEi 75 days; (C) Alport mice, ACEi 100 days; (D) Alport mice, Plac (untreated); (E) Alport mice, ACEi+Spiro 75 days; (F) Alport mice, ACEi-Spiro 100 days; (G) score for tubulointerstitial matrix accumulation ( $n=3$ for each group); $(\mathbf{H})$ score for glomerular matrix accumulation ( $n=3$ for each group); ${ }^{* * *} p<0.001 ;{ }^{* *} p<0.01{ }^{*} p<0.05$.

Accelerated scar-tissue formation due to Alport syndrome was determined by fibronectinstaining in each group (Figure 4). Compared to wild type mice, tubulointerstitial and glomerular scarring was severely and significantly increased in untreated Alport mice (Plac) (Figure 4A,D; $p<0.001$ ). Adding spironolactone on top of ramipril, significantly improved tubulointerstitial and glomerular at 75 days of age compared to ACEi treated mice $(p<0.001)$ and untreated mice $(p<0.01)$. Similar to matrix accumulation at day 100 (Figure $3 G)$, the significance of this antifibrotic effect at day 75 could not be maintained until day 100 (Figure 4C,F). In conclusion, dual RAAS-blockade appeared to have a greater antifibrotic effect on glomerular and tubulointerstitial scarring in AS than ACEi monotherapy. 

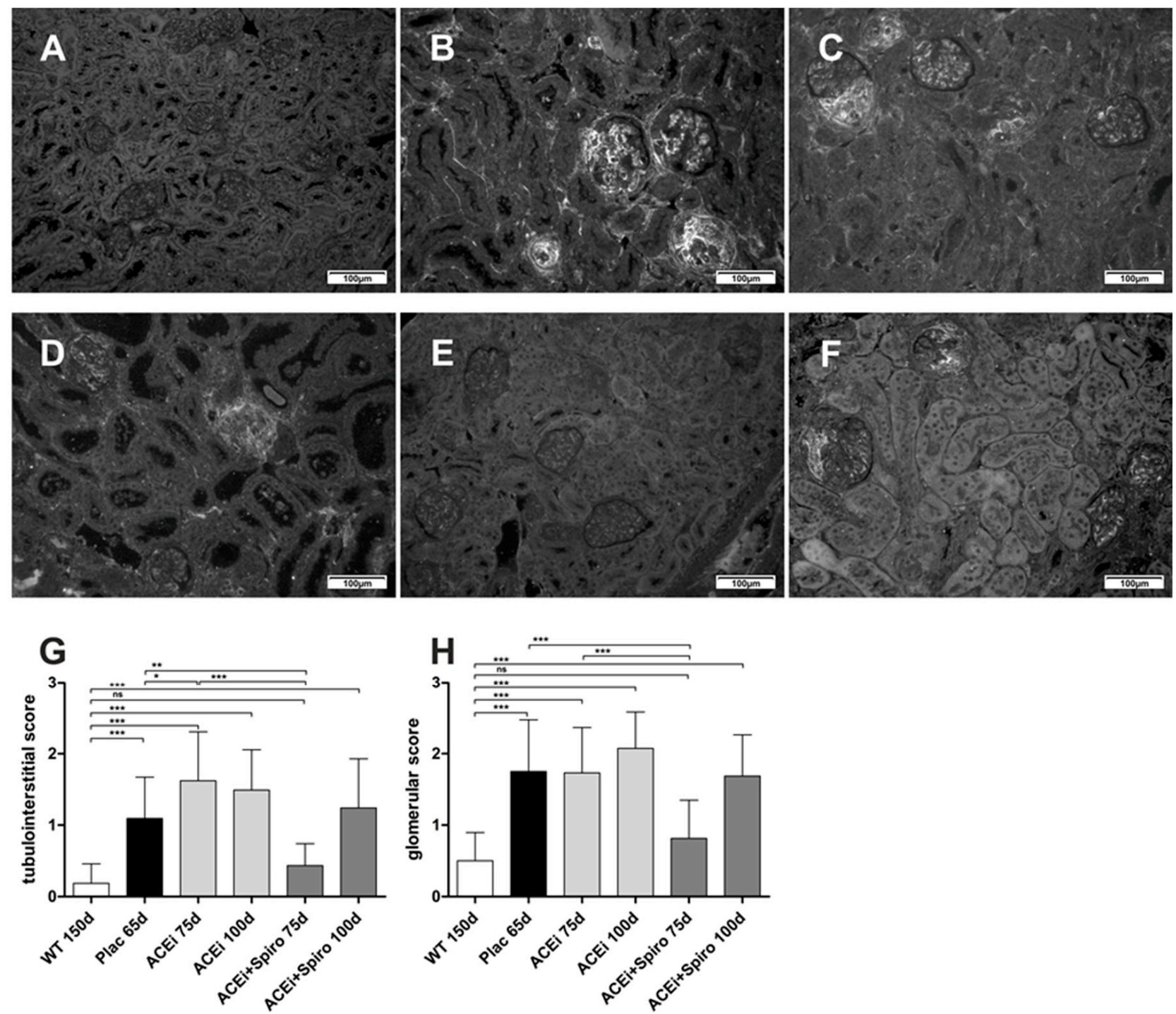

Figure 4. Therapeutic effect on scar tissue formation during pathogenesis of AS. Immune-fluorescence staining of fibronectin, magnification 200-fold. (A) wildtype control; (B) Alport mice, ACEi 75 days; (C) Alport mice, ACEi 100 days; (D) Alport mice, Plac (untreated); (E) Alport mice, ACEi+Spiro 75 days; (F) Alport mice, ACEi-Spiro 100 days; (G) score of tubulointerstitial fibrosis ( $n=3$ for each group); (H) score of glomerulosclerosis and fibrosis ( $n=3$ for each group); ${ }^{* * *} p<0.001 ;^{* *} p<0.01$; $* p<0.05$.

\subsection{Effect on Lifespan until Death Prior to End-Stage Renal Failure in Alport Mice}

In untreated COL4A3-/- mice (Plac), mean lifespan until death prior to end-stage renal failure (ESRF) was $66.5 \pm 3.7$ days (Figure 5). ACEi monotherapy improved lifespan to $101.7 \pm 19.1$ days. Despite superior BUN levels and improved antiproteinuric and antifibrotic potential of dual blockade (as shown in Figures 2-4), lifespan prior to endstage renal failure was not significantly improved by dual therapy (ACE+Spiro) with a mean survival of $89.3 \pm 17.6$ days (Figure 5). In conclusion, the improved kidney histology and kidney function parameters of ACEi+Spiro dual therapy were not reflected in better lifespan. 
A

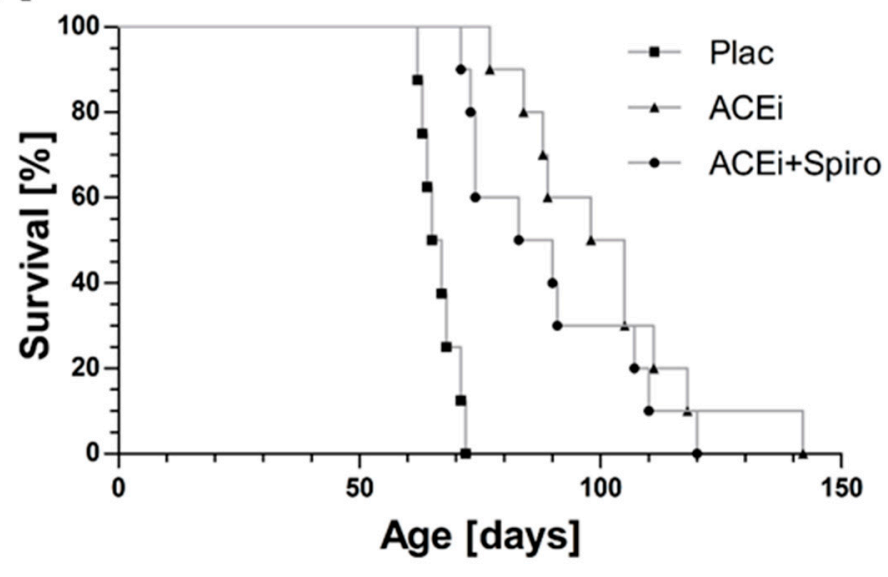

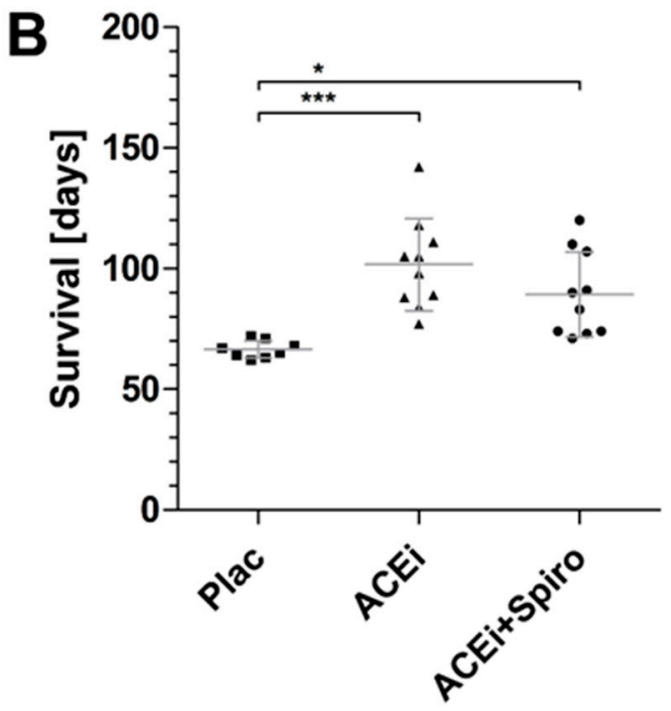

Figure 5. Therapeutic effect on timespan until end-stage renal failure. (A) Kaplan-Meier curves of lifespan in days until end-stage renal failure in untreated (Plac), ACEi treated and Alport mice with dual RAAS-blockade; (B) Box-blot of lifespan in untreated (Plac), ACEi treated and ACEi+Spiro treated Alport mice. $n=8$ for Plac, $n=10$ for ACEi and $n=10$ for ACEi+Spiro; ${ }^{* * *} p<0.001 ; * p<0.05$.

\subsection{Side-Effects of Dual RAAS-Blockade in Alport Mice}

The hyperkalemic side-effects of spironolactone under dual RAAS-blockade have been well described in humans, as well as the anti-androgen effects in males [18]. Similarly, the mean weight of male mice with dual therapy (ACEi+Spiro) increased significantly three weeks after initiation of spironolactone treatment $(25.3 \pm 2.5 \mathrm{~g}$ vs. ACEi monotherapy with $22.3 \pm 2.2$ g; Figure 6).

A

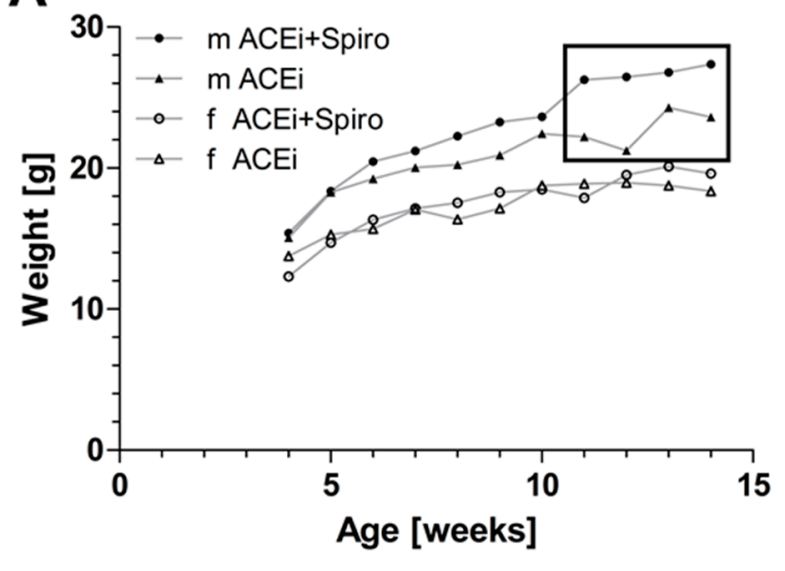

B

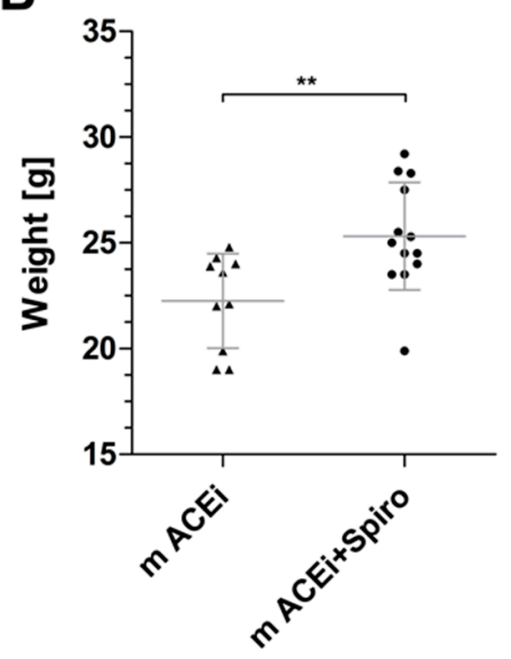

Figure 6. Anti-androgen side-effects of spironolactone on body weight. (A) Mean body weight of male and female Alport mice in the ACEi group vs. dual treated mice (ACEi+Spiro); (B) Individual measures of body weight of fully grown adult male mice in the ACEi group vs. dual treated males (ACEi+Spiro) as shown in the box in graph A $(n=10-13$ datapoints for each group). $\mathrm{m}=$ male; $\mathrm{f}=$ female; ${ }^{* *} p<0.01$.

The body weights of female mice were comparable in both treatment groups. The procedure of taking intra-cardial blood in non-heart-beating sacrificed mice is too susceptible to hyperkalemia in order to generate reliable values. Therefore, our study can only 
speculate about hyperkalemia as a possible side effect in dual treated Alport mice. In conclusion, dual RAAS-blockade by adding spironolactone on top of ACEi appeared to be associated with common and well-known side effects such as increasing body weight in male mice due to anti-androgen effect of spironolactone and, possibly, hyperkalemia.

\section{Discussion}

ACEis delay renal failure in patients with AS by many years [12]. However, over the long course of treatment, some of the antiproteinuric (and nephroprotective) effects of ACEis seem to weaken in a relevant number of patients. In the present study, increased levels of plasma aldosterone after long-term ACEi therapy, known as "aldosterone escape" [16,17], were hypothesized to be an important negative effect on disease progression in AS. Therefore, the present study investigates if the aldosterone antagonist spironolactone can counteract this loss of ACEi-efficacy in the Alport mouse model.

According to the low aldosterone levels (close to wildtype-controls) in our ACEi+Spiro group, dual therapy could be effective: dual therapy revealed in lower BUN levels, less proteinuria, less matrix accumulation, and less fibrosis than ACEi monotherapy. However, lifespan until end-stage renal failure was not improved. Recent data showed that the use of spironolactone in CKD patients is associated with a lower risk of ESRD, but also a higher risk of hyperkalemia-related hospitalization, indicating that hyperkalemia should be closely monitored during spironolactone therapy [18]. The incidence rate of severe hyperkalemia was about $6.5 \%$ in patients with an eGFR $<60 \mathrm{~mL} / \mathrm{min} / 1.73 \mathrm{~m}^{2}$, but zero in patients with eGFR $\geq 60 \mathrm{~mL} / \mathrm{min} / 1.73 \mathrm{~m}^{2}$ during dual RAAS-blockade with ACEi or ARB plus spironolactone. These data suggest that spironolactone-associated severe side effects (such as hyperkalemia) are more likely to occur in patients with CKD stages 3 and 4 [19]. In addition, dual RAAS-blockade with spironolactone resulted in a significant drop in blood pressure ( $-7 \mathrm{mmHg}$ diastolic, $-12 \mathrm{mmHg}$ systolic blood pressure), loss of eGFR $\left(-9.3 \mathrm{~mL} / \mathrm{min} / 1.73 \mathrm{~m}^{2}\right)$ and a significant decrease of albuminuria $(-57 \%)$ in diabetic patients after 24 weeks of treatment with spironolactone on top of ACEi or ARB [20]. Therefore, in our study, the beneficial antiproteinuric, antifibrotic effects of dual RAAS-blockade with spironolactone might not have resulted in improved lifespan prior to ESRF because of premature death due to severe side effects of dual RAAS-blockade such as hyperkalemia.

Hematuria is the earliest manifestation of Alport syndrome in the kidney, followed by microalbuminuria proceeding to overt proteinuria, impaired kidney function, finally leading to ESRF. Therefore, proteinuria and albuminuria are very important indicators of the severity of Alport syndrome and disease progression. In our study, adding spironolactone on top of ACEi decreased proteinuria and kept the level of proteinuria rather stable until late stages (at 100 days of age) compared to ACEi monotherapy. Further analysis of urine proteins showed that dual therapy did better in reducing high molecular weight proteins (as a sign for glomerular damage) and low molecular weight proteins (as a sign for tubular damage), suggesting that spironolactone had a protective effect on glomerular and tubular function. In parallel, tubular-interstitial and glomerular matrix accumulation were significantly reduced in ACEi+Spiro treated mice compared to ACEi monotherapy. As limitation of our study, the diuretic effect of spironolactone in the ACEi+Spiro group may have increased urine volume and reduced urinary protein concentration of spot urine. Glomerular and tubulointerstitial scar tissue formation was significantly reduced in dual treated mice compared to placebo and ACEi monotherapy. Therefore, our study showed a meaningful improvement in preserving kidney structure and function in the ACEi+Spiro group compared to ACEi-monotherapy in mice with Alport syndrome. Similar results of renoprotective effects from spironolactone add-on therapy have previously been reported in patients with albuminuria, including diabetic nephropathy [21,22]. In addition, patients with heart failure and renal dysfunction, spironolactone therapy has been shown to improve the two-year survival despite worsening eGFR and elevated potassium levels [23]. A small proof-of-concept study in five patients with AS, treated with spironolactone added 
after long term use of ACEi and ARBs, showed a significant reduction of the urinary protein/creatinine ratio at 3, 6, 12 and 18 months [24].

Elevated levels of plasma aldosterone significantly correlate with the decline of the glomerular filtration rate and higher risk of ESRF $[25,26]$. Our experiments were powered for the "primary end-point" end-stage renal failure, not for serum levels of aldosterone. This limits the informative value of the measurements of the aldosterone level, which were actually only intended as a further proof of concept. In our study with a very limited number of mice and aldosterone blood tests, ACEi+Spiro dual therapy resulted in constant low levels of serum aldosterone close to wildtype controls without CKD. Serum aldosterone levels were elevated in untreated Alport mice as well as in older Alport mice with long-lasting ACEi monotherapy, mimicking an "aldosterone-escape". Therefore, lower aldosterone levels in older Alport mice on dual therapy are the likely cause for improved kidney structure and function. However, Kim and coworkers reported that proteinuria reduction was not associated with the serum aldosterone concentration and add-on spironolactone treatment was more effective in the aldosterone non-escape group compared to the aldosterone escape group of CKD patients [19]. Recently, spironolactone was reported to be able to ameliorate endothelial dysfunction through intracellular oxidative stress attenuation in a 5/6 nephrectomy rat model [27]. It has been reported that spironolactone rescued renal dysfunction in obstructive jaundice rats by upregulating angiotensin-converting enzyme 2 expression, an important protective factor in diabetic nephropathy [28]. These data point to additional nephroprotective mechanisms of spironolactone despite its capability to reduce aldosterone levels, which should be explored in further studies $[29,30]$.

\section{Conclusions}

In our mouse model of Alport syndrome, spironolactone on top of ACEi is superior in preserving kidney function, kidney architecture, and delaying renal fibrosis. However, in our mouse model, the nephroprotective effect of dual RAAS-blockade was counteracted by premature death possibly due to a higher risk of serious side effects, therefore not improving the lifespan of Alport mice until ESRF. Therefore, dual RAAS-blockade by adding spironolactone could offer an effective therapy option for patients with Alport syndrome, who experience a loss of the antiproteinuric effect after long ACEi therapy due to an aldosterone-escape, which can be diagnosed by high aldosterone levels. Yet, the increased risk of potential side effects should be very seriously taken into account before starting dual RAAS-blockade. Dual RAAS-blockade requires close monitoring and strict safety measures, possibly including potassium-sparing diet or even potassium-binders added to the standard medication.

Author Contributions: Conceptualization, D.R., R.G. and O.G.; methodology, D.R., Y.Z. and N.S.; formal analysis, D.R., R.G. and O.G.; investigation, D.R., Y.Z. and N.S.; resources, O.G.; data curation, D.R.; writing —original draft preparation, D.R., Y.Z. and O.G.; writing—review and editing, N.S. and R.G.; visualization, D.R.; supervision, O.G.; funding acquisition, O.G. All authors have read and agreed to the published version of the manuscript.

Funding: This research was funded by the German Research Foundation DFG (GR 1852/6-1) to O.G. The authors gratefully acknowledge the support of K. C. Wong Education Foundation and DAAD.

Institutional Review Board Statement: Treatment protocols for the mice were approved by local German authorities (33.9-42502-04-14/1563; 31.07.2014) and supervised by veterinarians.

Informed Consent Statement: Not applicable.

Data Availability Statement: Data supporting reported results can be requested from the corresponding author.

Acknowledgments: The authors thank the staff from the animal house of the UMG facilities for their care.

Conflicts of Interest: The authors declare no conflict of interest. 


\section{References}

1. Zhou, J.; Leinonen, A.; Tryggvason, K. Structure of the human type IV collagen COL4A5 gene. J. Biol. Chem. 1994, 269, 6608-6614. [CrossRef]

2. Lemmink, H.H.; Mochizuki, T.; van den Heuvel, L.P.; Schröder, C.H.; Barrientos, A.; Monnens, L.A.; van Oost, B.A.; Brunner, H.G.; Reeders, S.T.; Smeets, H.J. Mutations in the type IV collagen alpha 3 (COL4A3) gene in autosomal recessive Alport syndrome. Hum. Mol. Genet. 1994, 3, 1269-1273. [CrossRef]

3. Boye, E.; Mollet, G.; Forestier, L.; Cohen-Solal, L.; Heidet, L.; Cochat, P.; Grünfeld, J.P.; Palcoux, J.B.; Gubler, M.C.; Antignac, C. Determination of the genomic structure of the COL4A4 gene and of novel mutations causing autosomal recessive Alport syndrome. Am. J. Hum. Genet. 1998, 63, 1329-1340. [CrossRef]

4. Flinter, F. Alport's syndrome. J. Med. Genet. 1997, 34, 326-330. [CrossRef]

5. Jais, J.P.; Knebelmann, B.; Giatras, I.; De Marchi, M.A.R.I.O.; Rizzoni, G.; Renieri, A.; Weber, M.; GROSS, O.; Netzer, K.O.; Flinter, F.; et al. X-linked Alport syndrome: Natural history in 195 families and genotype-phenotype correlations in males. J. Am. Soc. Nephrol. 2000, 11, 649-657. [CrossRef]

6. Bekheirnia, M.R.; Reed, B.; Gregory, M.C.; McFann, K.; Shamshirsaz, A.A.; Masoumi, A.; Schrier, R.W. Genotype-phenotype correlation in X-linked Alport syndrome. J. Am. Soc. Nephrol 2010, 21, 876-883. [CrossRef]

7. Wang, Y.; Sivakumar, V.; Mohammad, M.; Colville, D.; Storey, H.; Flinter, F.; Dagher, H.; Savige, J. Clinical and genetic features in autosomal recessive and X-linked Alport syndrome. Pediatr. Nephrol. 2014, 29, 391-396. [CrossRef]

8. Lee, J.M.; Nozu, K.; Choi, D.E.; Kang, H.G.; Ha, I.S.; Cheong, H.I. Features of Autosomal Recessive Alport Syndrome: A Systematic Review. J. Clin. Med. 2019, 8, 178. [CrossRef]

9. Zhang, Y.; Böckhaus, J.; Wang, F.; Wang, S.; Rubel, D.; Gross, O.; Ding, J. Genotype-phenotype correlations and nephroprotective effects of RAAS inhibition in patients with autosomal recessive Alport syndrome. Pediatr. Nephrol. 2021. online ahead of print. [CrossRef]

10. Gross, O.; Beirowski, B.; Koepke, M.L.; Kuck, J.; Reiner, M.; Addicks, K.; Smyth, N.; Schulze-Lohoff, E.; Weber, M. Preemptive ramipril therapy delays renal failure and reduces renal fibrosis in COL4A3-knockout mice with Alport syndrome. Kidney Int. 2003, 63, 438-446. [CrossRef]

11. Gross, O.; Schulze-Lohoff, E.; Koepke, M.L.; Beirowski, B.; Addicks, K.; Bloch, W.; Smyth, N.; Weber, M. Antifibrotic, nephroprotective potential of ACE inhibitor vs AT1 antagonist in a murine model of renal fibrosis. Nephrol. Dial. Transplant. 2004, 19, 1716-1723. [CrossRef]

12. Gross, O.; Licht, C.; Anders, H.J.; Hoppe, B.; Beck, B.; Tönshoff, B.; Höcker, B.; Wygoda, S.; Ehrich, J.H.; Pape, L.; et al. Early angiotensin-converting enzyme inhibition in Alport syndrome delays renal failure and improves life expectancy. Kidney Int. 2012, 81, 494-501. [CrossRef] [PubMed]

13. Webb, N.J.; Lam, C.; Shahinfar, S.; Strehlau, J.; Wells, T.G.; Gleim, G.W.; Le Bailly De Tilleghem, C. Efficacy and safety of losartan in children with Alport syndrome-results from a subgroup analysis of a prospective, randomized, placebo- or amlodipine-controlled trial. Nephrol. Dial. Transplant. 2011, 26, 2521-2526. [CrossRef] [PubMed]

14. Zhang, Y.; Wang, F.; Ding, J.; Zhang, H.; Liu, X.; Wang, S.; Xiao, H.; Yao, Y.; Liu, J.; Zhong, X.; et al. Long-term treatment by ACE inhibitors and angiotensin receptor blockers in children with Alport syndrome. Pediatr. Nephrol. 2016, 31, 67-72. [CrossRef]

15. ESCAPE Trial Group; Wühl, E.; Trivelli, A.; Picca, S.; Litwin, M.; Peco-Antic, A.; Zurowska, A.; Testa, S.; Jankauskiene, A.; Emre, S.; et al. Strict blood-pressure control and progression of renal failure in children. N. Engl. J. Med. 2009, 361, 1639-1650.

16. Schjoedt, K.J.; Andersen, S.; Rossing, P.; Tarnow, L.; Parving, H.H. Aldosterone escape during blockade of the renin-angiotensinaldosterone system in diabetic nephropathy is associated with enhanced decline in glomerular filtration rate. Diabetologia 2004, 47, 1936-1939. [CrossRef]

17. Bianchi, S.; Bigazzi, R.; Campese, V.M. Antagonists of aldosterone and proteinuria in patients with CKD: An uncontrolled pilot study. Am. J. Kidney Dis. 2005, 46, 45-51. [CrossRef] [PubMed]

18. Yang, C.T.; Kor, C.T.; Hsieh, Y.P. Long-term effects of spironolactone on kidney function and hyperkalemia-associated hospitalization in patients with chronic kidney disease. J. Clin. Med. 2018, 7, 459. [CrossRef] [PubMed]

19. Kim, H.Y.; Bae, E.H.; Ma, S.K.; Kim, S.W. Effects of spironolactone in combination with angiotensin-converting enzyme inhibitors or Angiotensin receptor blockers in patients with proteinuria. Kidney Blood Press. Res. 2014, 39, 573-580. [CrossRef] [PubMed]

20. Hase, M.; Babazono, T.; Ujihara, N.; Uchigata, Y. Comparison of spironolactone and trichlormethiazide as add-on therapy to renin-angiotensin blockade for reduction of albuminuria in diabetic patients. J. Diabetes Investig. 2013, 4, 316-319. [CrossRef]

21. Makhlough, A.; Kashi, Z.; Akha, O.; Zaboli, E.; Yazdanicharati, J. Effect of spironolactone on diabetic nephropathy compared to the combination of spironolactone and losartan. Nephrourol. Mon. 2014, 6, e12148. [CrossRef]

22. Selvaraj, S.; Claggett, B.; Shah, S.J.; Anand, I.; Rouleau, J.L.; O'Meara, E.; Desai, A.S.; Lewis, E.F.; Pitt, B.; Sweitzer, N.K.; et al. Prognostic value of albuminuria and influence of spironolactone in heart failure with preserved ejection fraction. Circ. Heart Fail. 2018, 11, e005288. [CrossRef]

23. Stubnova, V.; Os, I.; Grundtvig, M.; Atar, D.; Waldum-Grevbo, B. Spironolactone treatment and effect on survival in chronic heart failure patients with reduced renal function: A propensity-matched study. Cardiorenal Med. 2017, 7, 128-136. [CrossRef]

24. Kaito, H.; Nozu, K.; Iijima, K.; Nakanishi, K.; Yoshiya, K.; Kanda, K.; Krol, R.P.; Yoshikawa, N.; Matsuo, M. The effect of aldosterone blockade in patients with Alport syndrome. Pediatr. Nephrol. 2006, 21, 1824-1829. [CrossRef] 
25. Issa, N.; Ortiz, F.; Reule, S.A.; Kukla, A.; Kasiske, B.L.; Mauer, M.; Jackson, S.; Matas, A.J.; Ibrahim, H.N. The renin-aldosterone axis in kidney transplant recipients and its association with allograft function and structure. Kidney Int. 2014, 85, 404-415. [CrossRef]

26. Gant, C.M.; Laverman, G.D.; Vogt, L.; Slagman, M.C.; Heerspink, H.J.; Waanders, F.; Hemmelder, M.H.; Navis, G. Renoprotective RAAS inhibition does not affect the association between worse renal function and higher plasma aldosterone levels. BMC Nephrol. 2017, 18, 370. [CrossRef]

27. Wang, C.-C.; Lee, A.-S.; Liu, S.-H.; Chang, K.-C.; Shen, M.-Y.; Chang, C.-T. Spironolactone ameliorates endothelial dysfunction through inhibition of the AGE/RAGE axis in a chronic renal failure rat model. BMC Nephrol. 2019, 20, 351. [CrossRef]

28. Kong, E.-1.; Zhang, J.-m.; An, N.; Tao, Y.; Yu, W.-f.; Wu, F.-x. Spironolactone rescues renal dysfunction in obstructive jaundice rats by upregulating ACE2 expression. J. Cell Commun. Signal. 2019, 13, 17-26. [CrossRef]

29. Torra, R.; Furlano, M. New therapeutic options for Alport syndrome. Nephrol. Dial. Transplant. 2019, 34, 1272-1279. [CrossRef] [PubMed]

30. Yamamura, T.; Horinouchi, T.; Omori, T.; Sakakibara, N.; Aoto, Y.; Ishiko, S.; Nakanishi, K.; Shima, Y.; Nagase, H.; Takeda, H.; et al. Genotype-phenotype correlations influence the response to angiotensin-targeting drugs in Japanese patients with male X-linked Alport syndrome. Kidney Int. 2020, 98, 1605-1614. [CrossRef] 